\title{
Egstudios Gerenciales
}

Journal of Management and Economics for lberoamerica

Artigo de pesquisa

\section{Valores humanos como preditores de atitudes colaborativas e intenção de uso da plataforma de hospedagem do Airbnb}

Miriam Leite Farias*

Doutoranda em Administração, Programa de Pós-Graduação em Administração, Universidade Federal de Pernambuco - UFPE, Recife, Brasil. miriamlfarias@gmail.com

Maria de Lourdes de Azevedo Barbosa

Professora, Programa de Pós-Graduação em Hotelaria e Turismo, Universidade Federal de Pernambuco - UFPE, Recife, Brasil.

lourdesbarbosa@gmail.com

Bruna Lourena de Lima Dantas

Professora, Departamento de Administracão, Universidade Federal Rural do Semi-árido-UFERSA, Mossoró, Brasil.

Doutoranda em Administração, Universidade Federal da Paraíba, João Pessoa, Brasil.

brunalimadantas.adm@gmail.com

Bianca Gabriely Ferreira Silva

Doutoranda em Administração, Programa de Pós-Graduação em Administração, Universidade Federal de Pernambuco - UFPE, Recife, Brasil. biianca_ferreira@hotmail.com

Luana Alexandre Silva

Doutoranda em Administração, Programa de Pós-Graduação em Administração, Universidade Federal de Pernambuco - UFPE, Recife, Brasil. luanaalsilva@gmail.com

\section{Resumo}

0 presente estudo objetiva identificar o poder de predição dos valores humanos nas atitudes e na intenção comportamental em relação ao uso do consumo colaborativo. Para tanto, adotou-se uma abordagem quantitativa com a aplicação de um survey com 208 consumidores da plataforma de acomodacões Airbnb, os quais responderam questões sobre valores básicos fundamentados na teoria Funcionalista de valores humanos e atitudes e intenção comportamental. Os resultados apontam uma correlação significativa entre a subfunção de Experimentação e Atitudes relativas ao uso dos serviços, indicando que os valores que compõem essa subfunção teriam influência quanto às atítudes dos consumidores em relação ao serviço da plataforma. Dessa forma, tais resultados dão suporte à utilização dos valores humanos como facilitadores na promoção de comportamentos colaborativos.

Palavras-chave: consumo colaborativo; valores humanos; Airbnb

Los valores humanos como predictores de actitudes colaborativas y la intención de utilizar la plataforma de alojamiento de Airbnb

\section{Resumen}

Este estudio tuvo como objetivo identificar el poder predictivo de los valores humanos en las actitudes y la intención conductual con relación al consumo colaborativo. Para ello, se adoptó un enfoque cuantitativo con la aplicación de una encuesta a 208 consumidores de la plataforma de alojamiento Airbnb. Los encuestados respondieron preguntas sobre valores básicos, a partir de la teoría funcionalista de los valores humanos, las actitudes y la intención conductual. Los resultados apuntan a una correlación significativa entre la subfunción experimentación y actitudes relacionadas con el uso de los servicios; esto indica que los valores que componen esta subfunción influirían en las actitudes de los consumidores respecto al servicio de la plataforma. Por lo tanto, estos resultados apoyan el uso de valores humanos como facilitadores en la promoción de conductas colaborativas.

Palabras clave: consumo colaborativo; valores humanos; Airbnb.

Human values as predictors of collaborative attitudes and intention of using the Airbnb accommodation platform

\section{Abstract}

This study aimed to identify the predictive power of human values on attitudes and behavioral intention about to collaborative consumption. Therefore, a quantitative approach was adopted with the application of a survey with 208 consumers of the Airbnb accommodation platform. Respondents answered questions about basic values, based on the functionalist theory of human values, attitudes, and behavioral intention. The results indicate a significant correlation between the subfunction experimentation and attitudes related to the use of services, indicating that the values that make up this subfunction would influence the attitudes of consumers towards the platform service. Thus, these results support the use of human values as facilitators in the promotion of collaborative behaviors.

Keywords: collaborative consumption; human values; Airbnb.

* Autor para dirigir correspondência.

Classificações JEL: C83; D16.

Como citar: Farias, M. L., Barbosa, M. d. L. d. A., Dantas, B. L. d. L., Silva, B. G. F. e Silva, L. A. (2021). Valores humanos como preditores de atitudes colaborativas e intenção de uso da plataforma de hospedagem do Airbnb. Estudios Gerenciales, 37(160), 375-386. https://doi.org/10.18046/j. estger.2021.160.3991

DOI: https://doi.org/10.18046/j.estger.2021.160.3991

Recebido: 23-abr-2020

Aceito: 2-dez-2020

Publicado: 31-ago-2021 


\section{Introdução}

0 desenvolvimento de novas tecnologias e redes de comunicação vivenciado nas últimas décadas reforçou a ideia de coletividade entre as pessoas (Böcker e Meelen, 2017), permitindo que elas mesmas desenvolvam iniciativas e práticas de consumo alternativas, hoje denominadas de "consumo colaborativo" (Botsman e Rogers, 2010). Tendo como essência o compartilhamento de recursos, o consumo colaborativo se caracteriza como um sistema socioeconômico que prioriza o uso temporário de bens e serviços de maneira eficiente, ao invés da posse (Bostamn e Rogers, 2010; Nogueira-Pellizzoni e Baldanza, 2019). Hamari et al. (2016) definem o consumo colaborativo como atividades peer-to-peer (de pessoa para pessoa) realizadas para obter, dar ou compartilhar bens e serviços, coordenadas por meio de comunidades online.

0 avanço da Internet e das técnicas de Web 2.0 são fatores que influenciaram o estabelecimento desse tipo de consumo (Belk, 2007; 2014). Segundo Belk (2007), a Internet nos leva a um estágio de compartilhamento que proporciona igualdade de acesso a um fluxo de informação que é livre. As plataformas on-line tornam possível o compartilhamento em escala (Cohen e Kietzmann, 2014) e permitem que milhões de pessoas ao redor do mundo consigam acesso a formas de consumo mais convenientes e transparentes do que as tradicionais (Kathan; Matzler e Veider, 2016). Nesse contexto, empresas colaborativas precisam articular seus meios de interagir com o consumidor no ambiente digital para possibilitar uma percepção maior de valor para seus consumidores para que eles estejam dispostos a cocriar (Nogueira-Pellizzoni e Baldanza, 2019; Nadeem, Juntunena, Shirazib e Hajlic, 2019).

o comportamento dos indivíduos que se envolvem com modelos de consumo colaborativos é tema de interesse da academia e das organizações que atuam no mercado, pois a identificação das motivações, atitudes e valores que direcionam o consumo dessas pessoas pode contribuir para ampliar o entendimento sobre os hábitos de consumo presentes na contemporaneidade e auxiliar os profissionais do marketing a desenvolverem estratégias direcionadas para suas comunidades. Nesse sentido, estudos têm sido realizados no intuito de entender os fatores que determinam o consumo de práticas colaborativas (Hamari, Sjöklint e Ukkonen, 2016; Möhlmann, 2015; Piscicelli, Cooper e Fisher, 2015; Tussyadiah, 2015).

As características que influenciam o comportamento do consumidor se encontram divididas em duas vertentes: a primeira baseada em fatores demográficos (ex: gênero, idade, classe social) e a segunda, envolvendo variáveis psicográficas como personalidade, motivações, atitudes e valores. Ambas buscam prever e explicar os comportamentos dos indivíduos (Wells, 1975; Gunther e Furnham, 2015; Maoa et al., 2019). Nos estudos que abrangem os processos psicológicos e sociais em que ocorre a aquisição, uso e disposição de produtos, algumas pesquisas têm considerado o ponto de vista da Psicologia Social, que procura compreender comportamentos sociais, afetivos e cognitivos. A maioria dos pesquisadores visa compreender o porquê de as pessoas comprarem e como ocorre o processo de compra (Bagozzi, GurhanCanli e Priester, 2002).

Os fatores sociopsicológicos para compreender - comportamento podem ser classificados como motivacionais (ex: valores, atitudes e normas), individuais (ex: habilidades, status social), habituais (comportamentos passados) e externos (ex: publicidade, políticas de apoio) (Stern, 2000; Thøgerson, 2006). No consumo colaborativo, valores, atitudes, normas e comportamentos habituais são identificados como principais inibidores do processo de envolvimento das pessoas (Barnes e Mattson, 2016). Portanto, - conhecimento desses fatores poderá contribuir com estratégias que promovam o envolvimento do consumidor com atividades de consumo colaborativo.

Assim, o objetivo desta pesquisa é identificar o papel dos valores humanos como preditores de atitudes e intenção comportamental dos consumidores que utilizam os serviços colaborativos, exemplificados aqui pela plataforma peer-to-peer Airbnb, considerada uma expressão de consumo colaborativo (Wang et al., 2020).

Além dessa introdução, o artigo está dividido em mais quatro seções. Inicialmente, na revisão de literatura, são apresentados os construtos de valores humanos, considerando os fundamentos da teoria funcionalista proposta por Gouveia (2003), atitudes e intenção comportamental. 0 método quantitativo é então descrito e as análises dos resultados dos resultados trazidas nas seções seguintes. Por fim, nas conclusões, são apresentadas as considerações finais sobre a pesquisa, assim como, as suas limitações e sugestões futuras.

\section{Revisão de literatura}

\subsection{Consumo colaborativo}

Uma variedade de estudos sobre marketing e comportamento do consumidor busca examinar as razões subjacentes que levam as pessoas a consumirem da maneira como fazem, ou seja, os motivos inconscientes e conscientes que direcionam - comportamento. Considerando que as atitudes em relação ao consumo têm mudado nos últimos anos, devido à uma crescente preocupação com os impactos ambientais e sociais no planeta (Hamari, Sjökilnt e Ukkonen, 2016), compreende-se que seja necessário analisar as razões relacionadas ao envolvimento dos consumidores em atividades 
de compartilhamento e colaboração, abrangendo a complexidade dos determinantes que influenciam no seu comportamento.

Alguns estudos já se propuseram a investigar tais determinantes, como atitudes e motivações dos consumidores de serviços de consumo colaborativo (Bardhi e Eckhardt, 2012; Möhlmann, 2015; Tussyadiah, 2015; Böcker e Meelen, 2017; Farias, Silva e Barbosa, 2019). Böcker e Meelen (2017) ressaltaram que o compartilhamento de acomodações é geralmente motivado pelo ganho financeiro, ou seja, pela economia, assim como o compartilhamento de carros e caronas é mais direcionado pelas questões ambientais e nas plataformas de refeições destacamse as motivações sociais.

Com relação aos usuários e provedores, verificase também diferenças de motivações quando se considera a relação idade e renda. Grupos de jovens com renda menor são motivados a serem usuários e provedores por questões econômicas, e os jovens com renda alta e educação superior são motivados pelas questões de relacionamento social (Böcker e Meelen, 2017). Tussyadiah (2015) identificou que dentre as motivações que impulsionam o envolvimento no consumo colaborativo estão os fatores como sustentabilidade, comunidade e economia vistos também em Böcker e Meelen (2017), e como impedimentos de uso estão a falta de confiança, a falta de eficácia em relação à tecnologia e a falta de benefícios econômicos. A autora também sugere que o mercado da economia de compartilhamento no contexto de acomodação possui usuários com educação superior e renda elevada e os usuários são mais propensos à inovação do que os não usuários.

Destarte, Wang, Assaad e Filieri (2020) afirmam que o Airbnb e outras plataformas de economia compartilhada devem estar atentas aos aspectos sociais em sua plataforma de comércio, uma vez que seria considerado como atrativo pelos seus consumidores, especialmente para indivíduos com tendências pró-sociais, mais inclinados a quererem contribuir em economia compartilhada, fazendo parte de uma comunidade virtual. Esse papel social deve ser comunicado ao mercado-alvo, pois a utilidade do compartilhamento social se tornou valorizada, como também exercer um papel socialmente responsável.

Com relação ao construto de Valores Humanos na perspectiva de Consumo Colaborativo, foram encontrados quatro estudos, o de Piscicelli, Cooper e Fisher (2016) e o de Martin e Upham (2015), que se embasaram na teoria de Valores de Schwartz (1992, 1994, 2006), utilizando o Questionário de Valor de Retrato (PQV - Portrait Value Questionnaire) de Schwartz (2006), onde os respondentes apontam o quão similar se acham com as pessoas descritas nos retratos mostrados (portraits). 0 terceiro, de Ross e Hahn (2017), que buscou os efeitos do comportamento nos valores, atitudes e normas dos consumidores. E por fim, o de Arruda, Bandeira, Da Silva e Rebouças (2016), o qual foi aplicado no contexto brasileiro para analisar as relações entre os valores pessoais dos consumidores e o consumo colaborativo de bicicletas compartilhadas na cidade de Fortaleza.

No estudo de Piscicelli, Cooper e Fisher (2015), benevolência e universalismo aparecem como prioridades para os consumidores e os valores de autotranscendência e abertura à mudança se destacam em relação aos valores de autopromoção e conservação. A Tradição, entendida como o ato de preservar bens culturais, familiares e tradições religiosas, se revela um valor dificultador para o consumo colaborativo, já que esse modelo de consumo traz inovações que desafiam as formas tradicionais (Piscicelli, Cooper e Fisher, 2015).

Já para Martin e Upham (2015), o valor Tradição se mostra bem pontuado pelos consumidores, sendo também descartados os valores de autotranscendência e abertura à mudança. Para analisar o impacto dos valores dos indivíduos, os autores utilizaram a teoria de Schwartz sobre valores universais e a Teoria da Incorporação Coletiva de Valores de Chen, Lune e Queen (2013) para desenvolver um modelo conceitual, testado com indivíduos de grupos de reuso.

Em oposição ao entendimento de que os valores podem influenciar o comportamento, Ross e Hahn (2017) procuram entender os efeitos do consumo compartilhado (shared consumption) nos valores, atitudes e normas dos consumidores, utilizando da teoria do comportamento planejado de Ajzen (1991) e da teoria da Norma-valor-crença de Stern et al. (1999) para mensurar as mudanças nas variáveis, influências causais e efeitos habituais.

A análise da influência entre os valores pessoais dos consumidores no consumo de bicicletas compartilhadas, realizada por Arruda et al. (2016), revela que a economia de custos e tempo, a consciência socioambiental e crença no bem comum são motivadores de destaque para essa modalidade. Os valores pessoais de Autodeterminação e Estimulação que se referem à abertura à mudança são os que possuem maior destaque, sendo o perfil dos consumidores composto por usuários jovens e solteiros, com renda intermediária.

Após essa revisão de estudos envolvendo os fatores sociopsicológicos que motivam 0 envolvimento em atividades colaborativas, percebese que os construtos de valores humanos, atitudes e intenção comportamental no contexto do consumo colaborativo não foram analisados a partir da teoria funcionalista dos valores humanos de Gouveia (1998, 2003), a qual pode trazer contribuições para a discussão em função dos melhores resultados apresentados, tanto na adequação da 
hipótese de estrutura quanto no poder explicativo, mostrando correlação média com os indicadores comportamentais superior ao modelo proposto por Schawrtz (De Lima, 2012).

Sendo assim, pesquisas com esse tipo de proposta são necessárias, principalmente envolvendo o contexto brasileiro, em que a delimitação do tema consumo colaborativo ainda é recente.

\subsection{Os valores humanos sob a perspectiva funcionalista}

Os Valores Humanos são compreendidos como princípios guias na vida das pessoas (Rokeach, 1973; Gouveia, 2003), os quais podem variar de acordo com experiências pessoais, sociais e culturais do indivíduo (Xiao e Kim, 2009). Hofstede (1984) define os valores humanos como representações de necessidades sociais construídas a partir de seus estudos sobre valores específicos em diferentes culturas. Esses valores podem moldar o comportamento dos indivíduos (Sharma e Jha, 2017), dessa forma, são também observados em estudos do comportamento do consumidor.

Pesquisas recentes têm relacionado essa perspectiva vinda da Psicologia Social no comportamento do consumidor de modelos alternativos de consumo, como é o caso do consumo colaborativo (Piscicelli, Cooper e Fisher, 2015; Martin e Upham, 2015), do consumo sustentável (Sharma e Jha, 2017) e do consumo compartilhado (Ross e Hahn, 2017).

Para a Psicologia Social, os valores humanos representam os princípios guias na vida dos seres humanos (Rokeach 1968; Schultz e Zelezny, 1999; Gouveia, Fonsêca, Milfont e Fishcer, 2011) que transcendem objetos e situações específicas (Dose, 1997; Rokeach, 1973; Gouveia et al., 2011). Esses valores não entram em decadência ou ascensão, permanecem os mesmos, o que mudam são as prioridades valorativas dadas a cada um (Rokeach, 1973). Isso significa que há um número reduzido de valores que são resultantes de necessidades psicológicas e demandas sociais, pois são passados de geração a geração (Gouveia, 2013) e aprendidos por meio de um processo de socialização realizado por instituições sociais, como a família e a escola, afetando as atitudes dos indivíduos (Ros, 2006). Assim, os valores permanecem os mesmos em diferentes épocas, apresentando arranjos estruturais variados (Rokeach, 1973; Vione, 2012).

0 conhecimento dos valores dos consumidores pode trazer o desenvolvimento de estratégias de marketing eficazes para as organizações, pois eles permanecem intrínsecos nas pessoas durante muito tempo e influenciam na formação das suas atitudes em relação ao consumo.

Desde o final da década de 1980, o modelo dos tipos motivacionais criado por Schwartz e seus colaboradores tem sido uma grande referência nas pesquisas que relacionam valores humanos. Schwartz se inspirou em elementos dos trabalhos de Kluckhohn (1951), em que valores são entendidos como desejáveis, e nos fundamentos de Rokeach (1973). onde os valores possuem natureza motivacional, para propor uma estrutura de valores que atendam metas motivacionais dos indivíduos, buscando satisfazer as necessidades humanas. Em sua definição, os valores são metas desejáveis, que independente de situações específicas, guiam a seleção e avaliam comportamentos e eventos (Schwartz e Bilsky, 1987; Schwartz, 1992; Schwartz, 1994), exercendo um papel significativo nas relações entre grupos de pessoas à medida que se configuram como elementos cruciais dentro de uma cultura específica.

Gouveia (2003, p. 431) afirma que os valores humanos "emergem associados às experiências de socialização e dependem do contexto sociocultural de cada pessoa."

Além do modelo de tipos motivacionais de Schwartz, os modelos funcionais também têm sido usados para explicar e compreender diversos construtos da área da Psicologia, principalmente aqueles relacionados às pesquisas sobre atitudes (Maio e Olson, 2000). Apesar de pouca ênfase dos pesquisadores ao tema sobre funções (Gouveia, 2013; Gouveia et al., 2011; Allen, $\mathrm{Ng}$ e Wilson, 2002), é possível identificar na literatura duas funções valorativas principais, que são as que guiam as ações do indivíduo (Rokeach, 1973; Schwartz, 1992) e as que expressam as necessidades (Inglehart, 1977; Maslow, 1954). O reconhecimento dessas duas funções valorativas acarretou o desenvolvimento da Teoria Funcionalista dos Valores Humanos.

Ao comparar a teoria universal dos valores humanos de Schwartz e a teoria Funcionalista de Gouveia, com relação à hipótese de conteúdo e estrutura de poder preditivo das variáveis, De Lima (2012) verificou que ambos os modelos apresentaram correlações moderadas com indicadores comportamentais. No entanto, o modelo desenvolvido por Gouveia apresentou o índice de correlação média $(0,47$, dep $=0,14)$ superior ao de Schwartz 10,33 , $\mathrm{dp}=0,08)$.

Para a perspectiva funcionalista, Gouveia (2003, p. 433) define os valores como categorias de orientação que são adotadas pelos atores sociais com base nas necessidades humanas e precondições para satisfazêlas, e que podem variar em sua magnitude e seus elementos constituintes. Seu modelo de análise parte dos pressupostos de que a natureza do ser humano é benevolente (Maslow, 1954), então os valores são positivos e desejáveis (Kluckhohn, 1951); os valores constituem princípios guias da vida do indivíduo (Rockeach, 1973); possuem fundamento motivacional (Inglehart, 1977; Maslow, 1954) e são terminais pois representam metas individuais superiores (Rockeach, 1973) (Gouveia et al., 2011; Athayde, 2012). 
Rokeach (1973) identifica dois tipos de valores terminais: os valores sociais e os pessoais. Indivíduos guiados por valores pessoais lex: uma vida excitante, harmonia interiorl são autocentrados ou possuem foco intrapessoais, já aqueles guiados por valores sociais (ex: amizade verdadeira, um mundo de paz) são centrados na sociedade ou possuem foco interpessoais (Gouveia, Andrade, Milfont, Queiroga e Santos, 2003). Essa classificação também pode ser observada no trabalho de Hofstede (1984) sobre a ideia de individualismo e coletivismo. No entanto, há outros tipos de valores que não são nem totalmente sociais ou pessoais. É o caso de valores como estabilidade, maturidade e saúde (Gouveia, 2003; Gouveia et al. 2003; Mueller e Wornhoff, 1990) ou ainda, por exemplo, a ideia de valores motivacionais mistos (Schwartz, 1992).

Dessa forma, na teoria Funcionalista, não se segue o pensamento de Schwartz (1992) de que os valores são exclusivamente pessoais ou sociais, mas congruentes a ambos, ou seja, se situam entre os dois, permanecendo como referência central. Portanto, a primeira dimensão de guia de comportamento diferencia os valores de acordo com sua orientação, sendo ela pessoal, social ou central (Gouveia et al., 2014; Gouveia et al., 2015). Os valores pessoais são subdivididos em de experimentação e realização, os sociais, em normativos e de interação, e finalmente, os centrais, em valores de existência e suprapessoais (Gouveia, 1998, 2003).

Em seguida, a segunda dimensão diz respeito à expressão das necessidades dos indivíduos. De acordo com Maslow (1954) e Iglehart (1977). necessidades biológicas e sociais fazem parte do ser humano e são expressas por valores materialistas. Elas são conduzidas por normas subjetivas. De outro lado, os valores idealistas são expressos por princípios ainda mais abstratos e figuram após as necessidades básicas. A abertura à mudança e criatividade é observada mais fortemente em indivíduos que possuem estes valores, e que são mais independentes de bens materiais.

Assim, a segunda dimensão dos valores os diferencia de acordo com as necessidades humanas, sendo o tipo motivador materialista ou idealista (Gouveia et al., 2014; Gouveia et al., 2015).

As duas dimensões, guia de comportamento e expressão de necessidades, formam dois eixos principais que representam a estrutura espacial dos valores. 0 eixo horizontal condiz com as metas que orientam o comportamento (pessoal, social ou central) e o eixo vertical com o tipo motivador, as necessidades que os valores expressam (materialistas ou idealistas). 0 cruzamento entre os dois eixos gera um framework $3 \times 2$ com seis subfunções e valores básicos específicos (Gouveia et al., 2015; Gouveia, 1998, 2003). A Figura 1 apresenta uma descrição esquemática dessa interação.

\begin{tabular}{|c|c|c|c|c|}
\hline & \multicolumn{3}{|c|}{ Valores como padrão-guia de comportamentos } \\
\hline & & $\begin{array}{c}\text { Metas pessoais (o } \\
\text { indivíduo por si mesmo) }\end{array}$ & $\begin{array}{c}\text { Metas centrais (o } \\
\text { propósito geral da } \\
\text { vida) }\end{array}$ & $\begin{array}{l}\text { Metas sociais (o } \\
\text { individuo na } \\
\text { comunidade) }\end{array}$ \\
\hline \multirow{2}{*}{ 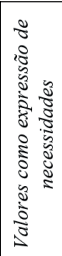 } & $\begin{array}{l}\text { Necessidades } \\
\text { idealistas (a vida } \\
\text { como fonte de } \\
\text { oportunidades) }\end{array}$ & $\begin{array}{c}\text { Experimentação } \\
\text { Emoção } \\
\text { Sexualidade } \\
\text { Prazer }\end{array}$ & $\begin{array}{l}\text { Suprapessoal } \\
\text { Beleza } \\
\text { Conhecimento } \\
\text { Maturidade }\end{array}$ & $\begin{array}{c}\text { Interativa } \\
\text { Afetividade } \\
\text { Apoio social } \\
\text { Convivência }\end{array}$ \\
\hline & $\begin{array}{l}\text { Necessidades } \\
\text { materialistas (a } \\
\text { vida como fonte } \\
\text { de ameaça) }\end{array}$ & $\begin{array}{c}\text { Realização } \\
\text { Exxito } \\
\text { Poder } \\
\text { Prestigio }\end{array}$ & $\begin{array}{l}\text { Existência } \\
\text { Estabilidade } \\
\text { Saúde } \\
\text { Sobrevivência }\end{array}$ & $\begin{array}{c}\text { Normativa } \\
\text { Obediência } \\
\text { Religiosidade } \\
\text { Tradição }\end{array}$ \\
\hline
\end{tabular}

Figura 1. Facetas, dimensões e valores básicos Fonte: adaptado de Gouveia et al. (2014).

Em suma, o cruzamento dessas duas dimensões gera seis subfunções valorativas: Experimentação, Suprapessoal, Interativa, Realização, Existência e Normativa. E cada uma dessas subfunções acomoda três valores básicos. Dessa forma, os valores humanos da teoria Funcionalista são mensurados por 18 valores básicos: emoção, sexualidade, prazer, beleza, conhecimento, maturidade, afetividade, apoio social, convivência, êxito, poder, prestígio, estabilidade, saúde, sobrevivência, obediência, religiosidade e tradição (Gouveia et al. 2014).

Tendo em vista os resultados alcançados de outros estudos no contexto do consumo colaborativo, sugerese que os valores das Subfunções de Experimentação, Interação e Existência teriam um poder preditivo maior nas atitudes e na intenção de uso frente às plataformas de consumo colaborativo.

\subsection{Atitudes e intenção comportamental}

0 conceito de Atitudes é um dos mais relevantes nas pesquisas sobre o consumidor e na psicologia social (Cacioppo, Gardner e Bernston, 1999). A discussão acerca do tema considera a atitude como a avaliação positiva ou negativa, favorável ou desfavorável sobre um objeto (Allen, Machleit e Kleine, 1992).

Dessa forma, as atitudes se tornam resultado de um conjunto de experiências vividas pelo indivíduo, informações a ele fornecidas por outros e a influência do mercado e da mídia. Na relação existente entre a atitude e o comportamento, a atitude precede e ajuda a formar o comportamento. No caso dos profissionais de marketing, o comportamento a ser analisado seria a tomada de decisão de compra (Katz, 1963).

Para Kotler (1998), as escolhas dos consumidores são influenciadas por cinco fatores psicológicos: motivação, percepção, aprendizagem, crenças e atitudes. Blackwell, Miniard e Engel (2001) afirmam que a atitude refere-se a gostos e desgostos do indivíduo que irão determinar suas intenções comportamentais. Já para Percy e Rossiter (1992), os efeitos relativos a uma propaganda seriam tudo aquilo que leva o receptor a uma particular resposta.

Para esse estudo, embasamo-nos no conceito de atitudes e intenção comportamental da teoria da 
Ação Racional (Fishbein e Ajzen, 1977), onde a atitude é o resultado do somatório da multiplicação de dois subcomponentes: as consequências resultantes percebidas da performance do comportamento (crenças comportamentais) e as avaliações dessas consequências (Lima e D’Amorim, 1986; Ajzen, 1991). Compreende-se que, quanto mais favorável for a atitude em relação ao comportamento, maior também será intenção em relação a ele (Ajzen e Fishbein, 1980).

Já as intenções comportamentais são compreendidas como disposições para a realização dos comportamentos (Lima e D'Amorim, 1986). As intenções podem ser definidas como a percepção dos indivíduos que geram um comportamento específico (Ajzen e Fishbein, 1977; Fishbein e Ajzen, 1977).

As intenções são usadas para identificar os fatores motivacionais que influenciam um determinado comportamento; são indicações de o quanto as pessoas estão dispostas e quanto esforço pretendem exercer para desempenhar um comportamento. Como regra geral, quanto mais forte for a intenção, maior será a probabilidade de execução do comportamento. Espera-se que as intenções influenciem a realização do comportamento na medida em que o indivíduo apresente controle comportamental (Ajzen, 1991, 2001).

Na teoria da Ação Racional, a intenção de realizar um determinado comportamento é determinada por dois conceitos precedentes: a atitude relativa ao comportamento, que refere-se ao grau em relação ao qual uma pessoa tem avaliação favorável ou desfavorável do comportamento em questão; e a norma subjetiva, um fator social que concerne à pressão social percebida para realizar ou não o comportamento (Ajzen, 1991, 2001).

\section{Procedimentos metodológicos}

Esta pesquisa utiliza métodos quantitativos para a mensuração e análise dos dados. Como estratégia de investigação, realizou-se um levantamento (survey) com 208 consumidores brasileiros da plataforma de hospedagem Airbnb. A amostra do estudo foi determinada pelo critério de selecionar pessoas que já utilizaram pelo menos uma vez os serviços da plataforma.

A coleta de dados da pesquisa foi conduzida por meio da Internet, o questionário foi disponibilizado em páginas digitais relativas a discussões sobre plataformas de consumo colaborativo, além de ser direcionado por mensagens aos participantes da página do Facebook Airbnb Brasil. A realização do pré-teste aconteceu em duas fases. A primeira caracterizou-se em uma avaliação de três especialistas com conhecimentos específicos em Comportamento do Consumidor e Métodos Quantitativos. E a segunda, em uma aplicação com uma amostra piloto para verificar a compreensão das questões pelos respondentes, considerando tanto o visual quanto a legibilidade do questionário.

0 instrumento de pesquisa trata-se de um questionário contendo três blocos de respostas. A primeira parte consistiu na verificação dos valores humanos de acordo com a teoria Funcionalista, para isso foi utilizado o Questionário de Valores Básicos (QVB) (Gouveia, Milfont, Fischer e Santos, 2008). $\mathrm{Na}$ segunda parte, foram medidas as atitudes e a intenção comportamental de uso dos serviços de Airbnb por parte dos respondentes da pesquisa. Essa parte foi elaborada com base na Teoria da Ação Racional (Fishbein e Ajzen, 1977; Ajzen e Fishbein, 1980) e outros estudos sobre consumo colaborativo (por exemplo, Hamari, Sjöklint e Ukkonen, 2016). A última parte buscou a obtenção dos dados sociodemográficos dos respondentes, abordando as variáveis de sexo, idade, local de residência, escolaridade, estado civil, renda mensal familiar e número de pessoas que vivem no mesmo domicílio.

Para a analisar os dados foi utilizado o software Statistical Package for the Social Sciences (SPSS) versão 25.0, auxiliando na tabulação. A análise de distribuição dos dados foi realizada para observar a suposição de normalidade da análise multivariada e, em seguida, realizou-se a análise da normalidade a partir da verificação visual do histograma e do teste de Kolmogorov-Smirnov (K-S). Foram utilizadas tanto estatísticas descritivas, como cálculo de frequências, médias e desvios padrões, quanto estatísticas inferenciais, como análise de regressão linear múltipla. Inicialmente, as estatísticas descritivas foram utilizadas para descrever a amostra. Posteriormente, a análise de correlação de Pearson foi utilizada para identificar a relação entre as variáveis com relação à parte inferencial. Por fim, realizou-se a análise de regressão linear múltipla para identificar o poder preditivo das subfunções valorativas em relação às variáveis dependentes de atitudes e intenção comportamental.

\section{Análise dos resultados}

Um total de 302 pessoas participaram desta pesquisa. No entanto, ao selecionarmos apenas aqueles que já utilizaram os serviços do Airbnb pelo menos uma vez na vida, 94 questionários foram excluídos da amostra, restando no final 208 questionários válidos. Dentre estes, $54,8 \%$ dos respondentes eram do sexo feminino e $45,2 \%$ do sexo masculino, com idade média de 29,9 (DP=9,10), variando de 19 a 60 anos. Com relação ao Estado civil, $70,2 \%$ deles eram solteiros e o nível de escolaridade foi considerado alto, uma vez que $39,9 \%$ possuem ensino superior completo e $42,3 \%$ algum tipo de pósgraduação lespecialização, mestrado, doutorado ou 
pós-doutoradol. No que se refere à renda familiar mensal, foram obtidas 182 respostas válidas. A média foi de $\mathrm{R} \$ 8.653,29$ ( $\mathrm{DP}=\mathrm{R} \$ 7.823,45)$, variando de $R \$ 1.200$ à $R \$ 70.000$. A Tabela 1 mostra o perfil sociodemográfico dos participantes.

Tabela 1. Perfil sociodemográfico dos respondentes

\begin{tabular}{lll}
\hline \multicolumn{2}{l}{ Características demográficas } & Amostra \\
\hline Sexo & Feminino & $54,80 \%$ \\
& Masculino & $45,20 \%$ \\
Idade & Média & 29,9 \\
& DP & 9,1 \\
Estado civil & Solteiro & $70,20 \%$ \\
Escolaridade & Superior completo & $39,90 \%$ \\
Renda familiar mensal (R\$) & Média & $8.653,29$ \\
& DP & $7.823,45$ \\
Frequência de uso & Utilizou apenas uma vez & $47,10 \%$ \\
\hline
\end{tabular}

Fonte: elaboração própria.

Observa-se que os participantes da pesquisa são jovens adultos, solteiros, com nível de escolaridade e renda mensal familiar considerados altos. As duas últimas características, escolaridade e renda, são semelhantes nos perfis encontrados em outros estudos com o tema consumo colaborativo (Böcker e Meelen, 2017; Arruda et al., 2016; Möhlmann, 2015).

\subsection{Análises da confiabilidade e validade das escalas}

Para esta pesquisa, entende-se que foi feita a validade de construto pois as escalas utilizadas foram inicialmente obtidas por meio da revisão de literatura de trabalhos que tiveram objetivos semelhantes. A escala de valores humanos proposta por Gouveia et al. (2008) foi adaptada para o meio on-line, enquanto as outras latitudes e intenção comportamental) foram elaboradas pelas autoras com base em estudos que utilizam a base teórica da teoria da Ação Racional, além de alguns estudos que tratam essas variáveis no contexto do consumo colaborativo.

Já para verificar a confiabilidade das escalas, foi realizado o cálculo do coeficiente de alfa de Cronbach. Primeiramente, buscou-se conhecer os índices de consistência interna lalfa de Cronbach, $\alpha$ ) das subfunções valorativas e, em seguida, dos construtos de atitudes e intenção comportamental. Tais resultados são apresentados na Tabela 2.

$\mathrm{Na}$ Tabela 2, percebe-se que os valores de alfa de Cronbach, considerando as seis subfunções valorativas, variam de 0,404 a 0,678 , sendo valores de a relativamente aceitáveis. Ao analisar o grupo de variáveis que formam o construto Atitudes, a consistência interna obtida foi de 0,766, também considerada aceitável. Quanto ao construto de Intenção Comportamental, a consistência interna foi igual a 0,728. Ou seja, por meio de uma consistência interna adequada, os itens utilizados nas escalas conseguem representar propriamente cada construto analisado.

Tabela 2. Índices de consistência interna das escalas

\begin{tabular}{lcc}
\hline Escalas & Quantidade de itens & alfa de Cronbach \\
\hline Subfunção & 3 & 0,473 \\
Experimentação & 3 & 0,404 \\
Subfunção Suprapessoal & 3 & 0,582 \\
Subfunção Interação & 3 & 0,671 \\
Subfunção Realização & 3 & 0,570 \\
Subfunção Existência & 3 & 0,678 \\
Subfunção Normativa & 4 & 0,766 \\
Atitudes & 4 & 0,728 \\
Intenção Comportamental & &
\end{tabular}

$\alpha=$ alfa de Cronbach.

Fonte: elaboração própria.

\subsection{Análise da dimensionalidade das escalas}

A questão da dimensionalidade das escalas de atitudes e intenção comportamental foi constatada a partir da técnica de Análise Fatorial Exploratória. A análise fatorial é essencial na avaliação da validade de conteúdo e da dimensionalidade da escala, pois fornece ao pesquisador uma avaliação das interrelações entre as variáveis (Hair et al., 2009).

Nessa análise, foram utilizados os testes estatísticos de Kaiser-Meyer-Olkin (KMO) e o teste de esfericidade de Bartlett. Com a utilização da Análise Fatorial Exploratória (AFE) foi possível criar fatores levando em consideração as variáveis originais. Assim, foram realizadas três análises fatoriais exploratórias, onde em cada uma foi obtido um fator para as variáveis analisadas. De acordo com a Tabela 3 , verifica-se que para todas as análises fatoriais o KMO foi acima de 0,5 e os testes de esfericidade de Bartllet foram significativos, assim a utilização da AFE neste caso é apropriada.

Tabela 3. Fatores gerados pela AFE

\begin{tabular}{lcc}
\hline & Atitudes & Intenção Comportamental \\
\hline KMO & 0,725 & 0,684 \\
$\mathrm{X}^{2}$ & 187,617 & 261,023 \\
$\mathrm{gl}$ & 6 & 6 \\
$\mathrm{p}$-valor & $<0,001^{* *}$ & $<0,001^{* *}$ \\
$\mathrm{~N}^{0}$ de itens & 4 & 4 \\
$\%$ da variância & 55,299 & 60,138
\end{tabular}

* Teste significante ao nível de 0,05. ${ }^{* *}$ Teste significante ao nível de 0,01. Fonte: elaboração própria.

Os fatores que compõem a Teoria da Ação Racional, que são Atitudes e Intenção Comportamental, possuem 4 variáveis cada e explicam $55,3 \%, 59,5 \%$ e $60,1 \%$ de suas variáveis respectivamente. Dessa forma, considerando os resultados das análises de dimensionalidade das escalas, julga-se que seja permitido realizar os testes posteriores. 
4.3 Correlação entre valores humanos, atitudes e intenção comportamental

Com o intuito de estabelecer em que medida e direção as variáveis se relacionam, calculou-se um $r$ de Pearson. Na Tabela 4, encontram-se as correlações entre os fatores gerados pela Análise Fatorial Exploratória e os fatores representativos das subfunções valorativas identificadas na teoria funcionalista. É possível perceber que não existem altas correlações entre as variáveis. A maior correlação observada foi entre o fator Atitudes e o fator Intenção Comportamental com um $r=0,554$. A falta de altas correlações entre os fatores das variáveis que representam as subfunções dos valores básicos é um resultado a ser considerado, pois não causará problemas com multicolinearidade.

Conforme pode ser observado na Tabela 4, a dimensão Atitudes obteve uma correlação significativa com Intenção Comportamental $(r=0,55$, $p<0,011$. Também foram observadas correlações significativas com todas as subfunções valorativas, com exceção da Suprapessoal $(r=0,12)$. No que diz respeito à Intenção Comportamental, observouse que essa se correlacionou positivamente com as subfunções Interação $(r=0,16)$, Suprapessoal $(r=0,14)$ e Normativa $(r=0,17)$, porém não apresentou correlação significativa com Experimentação ( $r=0,07)$, Existência $(0,10)$ e Realização $(r=0,11)$.

Assim, podemos inferir que os valores humanos presentes nas subfunções valorativas, com exceção da Suprapessoal, possuem uma relação positiva com as atitudes dos consumidores em relação ao uso dos serviços de Airbnb. Destacando-se a maior correlação positiva com a subfunção de Experimentação, e a menor com a subfunção Normativa.

Em consonância com Gouveia (2013), a subfunção de Experimentação dispõe de valores com exiguidade de satisfação e prazer (hedonismo), que indicam mudanças e inovações no status quo. Assim, as pessoas que detêm esses valores são, em geral, jovens, que são mais desprendidos com relação à metas e planos para o longo prazo (Gouveia et al., 2014; Gouveia et al., 2015). Dessa forma, os resultados sugerem que os usuários de plataformas de consumo colaborativo são geralmente jovens (Böcker e Meelen, 2017; Arruda et al., 2016).
No que diz respeito à subfunção Normativa, esta transmite necessidades de sobrevivência, porém o seu principal elemento está em torno de metas sociais. Os valores normativos simbolizam necessidade de Segurança e Controle, assim como as condições pré existentes para a necessidades básicas. Tais valores possuem orientação social com foco nas regras sociais, os indivíduos guiados por esses valores dão mais ênfase à manutenção da cultura e das normas. Bem como, evidenciam a obediência à autoridade (Gouveia et al., 2014; Gouveia et al., 2015). Considerando que o valor humano de Tradição também está presente nessa subfunção, esse achado corrobora com o que apontam Piscicelli, Cooper e Fisher (2015) sobre a Tradição- entendida como o ato de preservar bens culturais, familiares e tradições religiosas- se revelar como um valor dificultador para o consumo colaborativo.

\subsection{Análises de regressão linear múltipla}

No intuito de aprofundar as relações entre as variáveis, foram realizadas análises de regressão linear múltipla. A análise de regressão múltipla é uma técnica de dependência utilizada para analisar a relação entre uma variável dependente e duas ou mais variáveis independentes, denominadas variáveis preditoras. Seu principal objetivo é utilizar os valores conhecidos das variáveis independentes para prever os valores da variável dependente (Hair et al., 2009). Nesta pesquisa, a utilização da análise de regressão linear múltipla teve o propósito de identificar as relações das subfunções valorativas com atitudes e intenção comportamental dos consumidores. Foram realizadas duas análises de regressão múltipla: subfunções sobre atitudes e subfunções sobre intenção comportamental.

Com o objetivo de conhecer o poder preditivo das subfunções valorativas para explicar as atitudes relativas ao uso do Airbnb, decidiu-se por considerar todas as seis subfunções como variáveis independentes e o fator Atitudes como variável dependente, utilizando o método Entrada forçada (Enter). Na Tabela 5, é possível observar os resultados da análise de regressão.

Tabela 4. Correlação entre as subfunções valorativas, atitudes e intenção comportamental

\begin{tabular}{|c|c|c|c|c|c|c|c|c|}
\hline & Intenção & Atitude & Inter. & SupraP. & Experi. & Norm. & Exist. & Realiz. \\
\hline Interação & $0,161 *$ & $0,219^{* *}$ & 1 & & & & & \\
\hline Experimentação & 0,073 & $0,277^{* *}$ & $0,165^{*}$ & 0,126 & 1 & & & \\
\hline Normativa & $0,179 * *$ & $0,145^{*}$ & $0,415^{* *}$ & $0,291 * *$ & 0,074 & 1 & & \\
\hline Existência & 0,100 & $0,203^{* *}$ & $0,348^{* *}$ & $0,329 * *$ & 0,129 & $0,389 * *$ & 1 & \\
\hline
\end{tabular}

*Correlação significante ao nível de 0,05. ** Correlação significante ao nível de 0,01

Fonte: elaboração própria. 
Tabela 5. Regressão das Subfunções Valorativas sobre Atitudes

\begin{tabular}{|c|c|c|c|c|c|}
\hline $\begin{array}{l}\text { Valores } \\
\text { Básicos } \\
\end{array}$ & B & Beta & $\begin{array}{l}\text { Erro } \\
\text { padrão }\end{array}$ & Valor $\mathrm{t}$ & Sig. \\
\hline Constante & $-3,054$ & & 0,665 & $-4,595$ & 0,000 \\
\hline $\begin{array}{l}\text { Subfunção de } \\
\text { Experimentação }\end{array}$ & 0,197 & $0,194^{*}$ & 0,075 & 2,622 & 0,009 \\
\hline $\begin{array}{l}\text { Subfunção de } \\
\text { Interação }\end{array}$ & 0,117 & 0,115 & 0,078 & 1,498 & 0,136 \\
\hline $\begin{array}{l}\text { Subfunção } \\
\text { Suprapessoal }\end{array}$ & 0,025 & 0,020 & 0,092 & 0,272 & 0,786 \\
\hline $\begin{array}{l}\text { Subfunção de } \\
\text { Realização }\end{array}$ & 0,108 & 0,116 & 0,075 & 1,438 & 0,152 \\
\hline $\begin{array}{l}\text { Subfunção } \\
\text { Normativa }\end{array}$ & $-0,020$ & $-0,029$ & 0,059 & $-0,340$ & 0,734 \\
\hline $\begin{array}{l}\text { Subfunção de } \\
\text { Existência }\end{array}$ & 0,131 & 0,113 & 0,088 & 1,483 & 0,140 \\
\hline
\end{tabular}

B (unstandardized coefficients) = inclinação da reta de regressão; $\beta$ (standardized coefficients) = indicador da relação entre VD e VI: $\mathrm{R}^{2}=$ proporção de variância explicada. Valores estatisticamente significativos são apresentados em negrito. ${ }^{*} p<0,05$.

Fonte: elaboração própria.

A partir da Tabela 5, observa-se que Atitudes relativas ao uso do Airbnb foram preditas apenas pela subfunção de Experimentação $(\beta=0,194, p<0,05)$. Todas as outras variáveis não foram significativas ao nível de 5\%. Este modelo obteve uma força preditiva baixa $\left(R^{2}=0,124\right)$. Com relação à estimação do modelo, a estatística $F$ foi igual a 4,719, $p<0,05$, não rejeitando a hipótese de que o modelo está bem ajustado. Ou seja, é possível inferir que os valores presentes na subfunção de Experimentação têm poder preditivo em relação às atitudes de uso dos serviços do Airbnb.

Como mencionado anteriormente, a subfunção de Experimentação possui tipo motivador idealista e seus valores contribuem para a promoção de inovações nas estruturas de organizações sociais, sendo endossados principalmente por jovens. Essa subfunção é composta pelos valores de emoção, que representa a busca por excitabilidade e experiências perigosas; prazer, que visa o interesse de aproveitar a vida no sentido amplo, desfrutar os prazeres ao máximo; e sexualidade, que representa a necessidade de sexo.

Talvez esse achado se relacione à dimensão de abertura a mudanças da teoria de Schwartz (1992, 1994). Tanto o trabalho de Piscicelli, Cooper e Fisher (2015) quanto o de Martin e Upham (2015) apontam que consumidores engajados em atividades de consumo colaborativo pontuam mais em valores de autotranscedência e abertura a mudanças. Assim como, com o que foi trazido por Tussyadiah (2015), sobre os usuários de plataformas de acomodações serem mais inovadores que os não usuários.

Posteriormente, no intuito de identificar o poder preditivo dos valores humanos sobre o uso de serviços do Airbnb, foi realizada uma análise de regressão múltipla com as subfunções valorativas sobre a variável de Intenção comportamental.
Tabela 6. Regressão das Subfunções valorativas sobre Intenção Comportamental

\begin{tabular}{|c|c|c|c|c|c|}
\hline $\begin{array}{l}\text { Valores } \\
\text { Básicos }\end{array}$ & $B$ & Beta & $\begin{array}{l}\text { Erro } \\
\text { padrão }\end{array}$ & Valor $\mathrm{t}$ & Sig. \\
\hline Constante & $-1,507$ & & 0,693 & $-2,175$ & 0,031 \\
\hline $\begin{array}{l}\text { Subfunção de } \\
\text { Experimentação }\end{array}$ & 0,017 & 0,017 & 0,078 & 0,221 & 0,826 \\
\hline $\begin{array}{l}\text { Subfunção de } \\
\text { Interação }\end{array}$ & 0,085 & 0,083 & 0,082 & 1,037 & 0,301 \\
\hline $\begin{array}{l}\text { Subfunção } \\
\text { Suprapessoal }\end{array}$ & 0,098 & 0,077 & 0,096 & 1,024 & 0,307 \\
\hline $\begin{array}{l}\text { Subfunção de } \\
\text { Realização }\end{array}$ & 0,024 & 0,026 & 0,078 & 0,304 & 0,761 \\
\hline $\begin{array}{l}\text { Subfunção } \\
\text { Normativa }\end{array}$ & 0,075 & 0,108 & 0,061 & 1,239 & 0,217 \\
\hline $\begin{array}{l}\text { Subfunção de } \\
\text { Existência }\end{array}$ & $-0,012$ & $-0,010$ & 0,090 & $-0,129$ & 0,897 \\
\hline
\end{tabular}

$\mathrm{B}$ (unstandardized coefficients) = inclinação da reta de regressão; $\beta$ (standardized coefficients) = indicador da relação entre VD e VI; $\mathrm{R}^{2}$ = proporção de variância explicada. Valores estatisticamente significativos são apresentados em negrito. ${ }^{*} p<0,05$.

Fonte: elaboração própria.

Com a análise, as subfunções valorativas mostraram não ser boas preditoras para a variável Intenção Comportamental. 0 modelo, segundo a estatística $F$, não está bem ajustado e sua força preditiva é muito baixa $\left(R^{2}=0,045\right)$. Ao observar a Tabela 6, é possível perceber que nenhuma variável foi significativa $(p<0,05)$, com exceção do intercepto. Assim, apesar de ter influência nas atitudes dos consumidores, os valores humanos da teoria Funcionalista não apresentam poder preditivo quanto à intenção de uso dos serviços do Airbnb diretamente.

\section{Conclusões do estudo}

O consumo colaborativo é um modelo socioeconômico proveniente do desenvolvimento de tecnologias em redes e da interação entre as pessoas. Essa forma alternativa de consumo busca geralmente intensificar o uso de recursos ociosos e reutilizar bens que não são mais desejados por seus donos.

Acredita-se que os valores dos consumidores podem consideravelmente dificultar ou contribuir para a aceitação, adoção e difusão de modelos de negócios baseados na ideia de colaboração e compartilhamento. Com isso, alguns autores investigaram a relação entre valores específicos e 0 engajamento em atividades de consumo colaborativo. No entanto, esses autores utilizam a teoria de valores humanos de Schwartz (1992), ou variações dela.

A principal contribuição deste trabalho foi utilizar a perspectiva da teoria Funcionalista de Gouveia (2003) para identificar o poder de predição dos valores humanos nas atitudes e intenção comportamental do uso de um serviço de hospedagem colaborativo. Para isso, foi deter- 
minado que a intenção comportamental seria a intenção de uso dos serviços do Airbnb, uma plataforma de aluguel de acomodações.

Os resultados das análises mostram que a dimensão Atitudes obteve correlações significativas com todas as subfunções valorativas, com exceção da Suprapessoal. A correlação mais alta observada foi com a subfunção Experimentação. Essa subfunção também foi a mais destacada quando realizada a regressão linear múltipla das subfunções valorativas sobre Atitudes.

A subfunção de Experimentação é caracterizada pela busca do prazer e seus valores básicos contribuem para a promoção de inovações. Acreditase que essa subfunção tem semelhanças com a dimensão de abertura a mudanças da teoria proposta por Schwartz (1992, 1994). Consumidores engajados em atividades de consumo colaborativo pontuam mais em valores de autotranscendência e abertura a mudanças (Piscicelli, Cooper e Fisher, 2015; Martin e Upham, 2015; Arruda et al., 2016).

Dessa forma, tomando para análise essa relação positiva, os profissionais responsáveis pelo marketing poderiam direcionar suas campanhas publicitárias específicas para alcançar valores relacionados à experimentação de novidades e maior abertura à mudança. Nesse sentido, o Airbnb se diferencia ao oferecer serviços inovadores aos seus usuários. Uma das inovações que podem ser pontuadas é a opção de realizar atividades atrativas como dança, aulas de culinária, passeios, aulas de yoga na cidade em que os hóspedes se hospedarão. Assim eles podem conviver com os moradores locais e gerar experiências de alto valor emocional.

Quanto às limitações do estudo, considerando a natureza não probabilística da amostra, não se pode generalizar os resultados encontrados para todos os usuários do Airbnb. Outro ponto a ser observado é que apesar do baixo poder preditivo dos valores humanos da teoria Funcionalista em relação à intenção de uso dos serviços do Airbnb, acreditase que essa abordagem ainda seja mais adequada para o contexto brasileiro, por ter sido desenvolvida localmente, comparada a dos valores motivacionais de Schwartz. Além disso, ao comparar as duas, a teoria Funcionalista apresenta melhores resultados tanto na adequação da hipótese de estrutura quanto no poder explicativo, mostrando maior correlação média com os indicadores comportamentais. Outro fator importante é que, na teoria de Schwartz 1992, 1994), faltam valores relativos à existência do indivíduo, os quais são colocados por Gouveia (2003).

Assim, para melhor verificar a eficácia da teoria Funcionalista sob a teoria Motivacional de Schwartz, sugere-se que, em estudos futuros, primeiramente, sejam utilizadas amostras maiores e mais heterogêneas. Seria interessante também considerar os dois lados da transação, de quem oferece o serviço e de quem o recebe, e ampliar a pesquisa para diferentes plataformas de consumo colaborativo, não apenas o Airbnb.

\section{Conflito de interesses}

Os autores declaram não haver conflito de interesses.

\section{Referências}

Ajzen, H., \& Fishbein, M. (1980). Understanding attitudes and predicting social behavior. New Jersey: Prentice-Hall.

Ajzen, I. (1991). The theory of planned behavior. Organizational Behavior and Human Decision Processes, 50(2), 179-211. https://doi.org/10.1016/0749-5978(91)90020-T

Ajzen, I., \& Fishbein, M. (1977). Attitude-behavior relations: A theoretical analysis and review of empirical research. Psychological Bulletin, 84(5), 888-918. https://doi.org/10.1037/0033-2909.84.5.888

Ajzen, I. (2001). Nature and operation of attitudes. Annual Review of Psychology, 52(1), 27-58. https://doi.org/10.1146/annurev.psych.52.1.27

Allen, C. T., Machleit, K. A. \& Kleine, S. S. (1992). A comparison of attitudes and emotions as predictors of behavior at diverse levels of behavioral experience. Journal of consumer research, 18(4), 493504. https://doi.org/10.1086/209276

Allen, M. W., Ng, S. H. \& Wilson, M. (2002). A functional approach to instrumental and terminal values and the value-attitude-behaviour system of consumer choice. European Journal of Marketing, 36, 111-135. https://doi.org/10.1108/03090560210412728

Arruda, H. R., Bandeira, E. L., Da Silva, Á. L. L. \& Rebouças, S. M. D. P. (2016). Consumo Colaborativo e Valores Pessoais: 0 Caso da Bicicleta Compartilhada. Revista Brasileira de Marketing, 15(5), 683-698. https://doi.org/10.5585/remark.v15i5.3370

Athayde, R. A. A. (2012). Medidas implícitas de valores humanos: elaboração e evidências de validade. Dissertação de Mestrado em Psicologia Social. Universidade Federal da Paraíba. Brasil.

Bagozzi, R., Gurhan-Canli, Z. \& Priester, J. (2002). The social psychology of consumer behaviour. London: McGraw-Hill Education.

Bardhi, F. \& Eckhardt, G. M. (2012). Access-based consumption: the case of car sharing. Journal of Consumer Research, 39(4), 881-898. https://doi.org/10.1086/666376

Barnes, S. J. \& Mattsson, J. (2016). Understanding current and future issues in collaborative consumption: A four-stage Delphi study. Technological Forecasting and Social Change, 104, 200-211. https://doi.org/10.1016/j.techfore.2016.01.006

Belk, R. (2007). Why not share rather than own? The Annals of the American Academy of Political and Social Science, 611(1), 126-140. https://doi.org/10.1177/0002716206298483

Belk, R. (2014). You are what you can access: Sharing and collaborative consumption online. Journal of Business Research, 67(8), 15951600. https://doi.org/10.1016/j.jbusres.2013.10.001

Blackwell, R., Miniard, P. \& Engel, J. (2001). Consumer behavior. Worth, Texas: Dryden Press, Harcourt College Publishers.

Böcker, L., \& Meelen, T. (2017). Sharing for people, planet or profit? Analysing motivations for intended sharing economy participation. Environmental Innovation and Societal Transitions, 23, 28-39. https://doi.org/10.1016/j.eist.2016.09.004

Botsman, R. \& Rogers, R. (2010). What's mine is yours: The rise of collaborative consumption. New York: HarperCollins.

Cacioppo, J. T., Gardner, W. L. \& Berntson, G. G. (1999). The affect system has parallel and integrative processing components: Form follows function. Journal of personality and Social Psychology, 76(5), 839-855. https://doi.org/10.1037/0022-3514.76.5.839

Chen, K. K., Lune, H. \& Queen, E. L. (2013). How values shape and are shaped by nonprofit and voluntary organizations: The current state 
of the field. Nonprofit and voluntary sector quarterly, 42(5), 856-885. https://doi.org/10.1177/0899764013480273

Cohen, B. \& Kietzmann, J. (2014). Ride on! Mobility business models for the sharing economy. Organization e Environment, 27(3), 279296. https://doi.org/10.1177/1086026614546199

Dose, J. J. (1997). Work values: an integrative framework and illustrative application to organizational socialization. Journal of Occupational and Organizational Psychology, 70, 219-240. https://doi.org/10.1111/j.2044-8325.1997.tb00645.x

Farias, M. L., Silva, A. L \& Barbosa, M. L. A. (2019). Motivos del no uso de los servicios de hospedaje compartido ofrecidos por las plataformas peer to peer. Estudios y Perspectivas en Turismo. 28, $1104-1121$

Fishbein, M., \& Ajzen, I. (1977). Belief, attitude, intention, and behavior: An introduction to theory and research. Philosophy and Rhetoric, 10(2), 130-132.

Gouveia, V. V. (1998). La naturaleza de los valores descriptres del individualismo y del colectivismo: una comparación intra e intercultural. Tese de Doutorado. Departamento de Psicologia Social, Universidade Complutense de Madrid, España.

Gouveia, V. V. (2003). A natureza motivacional dos valores humanos: evidências acerca de uma nova tipologia. Estudos de Psicologia, 8(3), 431-443. https://doi.org/10.1590/S1413-294X2003000300010

Gouveia, V. V. (2013). Teoria funcionalista dos valores humanos: fundamentos, aplicações e perspectivas. São Paulo: Casa do Psicólogo.

Gouveia, V. V., Andrade, J. M. D., Milfont, T. L., Queiroga, F. \& Santos, W. S. D. (2003). Dimensões normativas do individualismo e coletivismo: é suficiente a dicotomia pessoal vs. social? Psicologia: reflexão e crítica, 16(2), 223-234. https://doi.org/10.1590/S0102-79722003000200002

Gouveia, V. V., Milfont, T. L., Fischer, R. D. \& Santos, W. S. (2008). Teoria funcionalista dos valores humanos. In Teixeira, M. L. M. (Coord.), Valores humanos e gestão: Novas perspectivas (pp. 47-80). São Paulo: Editora Senac.

Gouveia, V. V., Fonsêca, P. N., Milfont, T. L. \& Fishcer, R. (2011). Valores humanos: contrinuições e perspectivas teóricas. In: Torres, C. V. e Neiva, E. R. (Eds.), A psicologia social: principais temas e vertentes (pp. 296-313). Porto Alegre, RS: ArtMed.

Gouveia, V. V., Milfont, T. L. \& Guerra, V. M. (2014). Functional theory of human values: testing its content and structure hypotheses. Personality and Individual Differences, 60 (1), 41-47. https://doi.org/10.1590/S1678-69712009000300004

Gouveia V. V., Milfont, T. L., Vione, K. C. \& Santos, W. S. (2015). Guiding actions and expressing needs: on the psychological functions of values. Psykhe, 24(2), 1-14. https://doi.org/10.7764/psykhe.24.2.884

Gunter, B. \& Furnham, A. (2015). Consumer Profiles (RLE Consumer Behaviour): An Introduction to Psychographics. London: Routledge.

Hair, J. F., Black, W. C., Babin, B. J., Anderson, R. E. \& Tatham, R. L. (2009). Análise multivariada de dados. Porto Alegre: Bookman.

Hamari, J., Sjöklint, M. \& Ukkonen, A. (2016). The sharing economy: Why people participate in collaborative consumption. Journal of the association for information science and technology, 67(9), 20472059. https://doi.org/10.1002/asi.23552

Hofstede, G. (1984). Culture's consequences: international differences in word-related values. Newbury Park, CA: Sage Publications.

Inglehart, R. (1977). The silent revolution: changing values and political styles among western publics. Princeton, NJ: Princeton University Press.

Kathan, W., Matzler, K. \& Veider, V. (2016). The sharing economy: Your business model's friend or foe? Business Horizons, 59(6), 663-672. https://doi.org/10.1016/j.bushor.2016.06.006

Katz, D. (1963). Determinants of attitude arousal and attitude change. Public Opinion Quarterly, 24, 176-192.

Kluckhohn, C. (1951). Los valores y las orientaciones de valor en la teoría de la acción. In: Parsons, T. e Shils, E.A. (Orgs.). Hacia una teoría general de la acción (pp. 435-485). Buenos Aires: Editorial Kapelusz.
Kotler, P. (1998). Administração de marketing: análise, planejamento, implementação e controle (5. ed.). São Paulo: Atlas.

De Lima, T. J. S. D. (2012). Modelos de valores de Schwartz e Gouveia: comparando conteúdo, estrutura e poder preditivo. Dissertação de Mestrado. Universidade Federal da Paraíba. Departamento de Psicologia. Brasil.

Lima, V. M. L. M. \& D'Amorim, M. A. (1986). A relação atitudecomportamento à luz da Teoria da Ação Racional. Arquivos Brasileiros de Psicologia, 38(1), 133-142.

Maio, G. R. \& Olson, J. M. (2000). Why we evaluate: functions of attitudes. Mahwah, NJ: Lawrence Erlbaum.

Maoa, Z., Jonesa, M. F., Lib, M., Weic, W. \& Lyud, J. (2019). Sleeping in a stranger's home: A trust formation model for Airbnb. Journal of Hospitality and Tourism Management, 42, 67-76. https://doi.org/10.1016/j.jhtm.2019.11.012

Martin, C. \& Upham, P. (2015). Grassroots social innovation and the mobilisation of values in collaborative consumption: a conceptual model. Journal of Cleaner Production, 134, 204-213. https://doi.org/10.1016/j.jclepro.2015.04.062

Maslow, A. (1954). Motivation and personality. New York: Harper and Row.

Möhlmann, M. (2015). Collaborative consumption: determinants of satisfaction and the likelihood of using a sharing economy option again. Journal of Consumer Behaviour, 14(3), 193-207. https://doi.org/10.1002/cb.1512

Mueller, D. J. \& Wornohoff, S. A. (1990). Distinguishing personal and social values. Educational and Psychological Measurement, 50, 691699. https://doi.org/10.1590/S1678-69712009000300005

Nadeema, W., Juntunena, M., Shirazib, F. \& Hajlic, N. (2019). Consumers' value co-creation in sharing economy: The role of social support, consumers' ethical perceptions and relationship quality. Technological Forecasting e Social Change, 151, 119786. https://doi.org/10.1016/j.techfore.2019.119786

Nogueira-Pellizzoni, L. \& Baldanza, R. F. (2019). Co-creation in conventional and collaborative businesses. Estudios Gerenciales, 35(151), 95-108. https://doi.org/10.18046/j.estger.2019.151.3055

Percy, L. \& Rossiter, J. R. (1992). Advertising stimulus effects: A review. Journal of Current Issues e Research in Advertising, 14(1), 75-90. https://doi.org/10.1080/10641734.1992.10504982

Piscicelli, L., Cooper, T. \& Fisher, T. (2015). The role of values in collaborative consumption: insights from a product-service system for lending and borrowing in the UK. Journal of Cleaner Production, 97, 21-29. https://doi.org/10.1016/j.jclepro.2014.07.032

Rokeach, M. (1973). The nature of human values. New York: The Free press.

Rokeach, M. (1968). Beliefs, attitudes and values; a theory of organization and change. San Francisco: Jossey-Bass.

Ros, M. (2006). Valores, atitudes e comportamento: uma nova visita a um tema clássico. In: Ros, M. and Gouveia, V.V. (Eds.), Psicologia social dos valores humanos: desenvolvimentos teóricos, metodológicos e aplicados (pp. 87-114). São Paulo: Senac.

Ross, D. \& Hahn, R. (2017). Does shared consumption affect consumers' values, attitudes, and norms? a panel study. Journal of Business Research, 77, 113-123. https://doi.org/10.1016/j.jbusres.2017.04.011

Schultz, P. W. \& Zelezny, L. (1999). Values as predictors of environmental attitudes: evidence for consistency across 14 countries. Journal of Environmental Psychology, 19, 255-265. https://doi.org/10.1006/jevp.1999.0129

Schwartz, S. H. (1992). Universals in the content and structure of values: Theoretical advances and empirical tests in 20 countries. Advances in experimental social psychology, 25(1), 1-65. https://doi.org/10.1016/S0065-2601 (08) 60281-6

Schwartz, S. H. (1994). Are there universal aspects in the structure and contents of human values? Journal of social issues, 50(4), 1945. https://doi.org/10.1111/j.1540-4560.1994.tb01196.x

Schwartz, S. H. (2006). Há aspectos universais na estrutura e no conteúdo dos valores humanos? In Psicologia social dos valores 
humanos: Desenvolvimentos teóricos, metodológicos e aplicados (pp. 55-85). São Paulo: Senac.

Schwartz, S. H \& Bilsky, W. (1987). Toward a universal psychological structure of human values. Journal of Personality and Social Psychology, 53, 550-562. https://doi.org/10.1037/0022-3514.53.3.550

Sharma, R., \& Jha, M. (2017). Values influencing sustainable consumption behaviour: Exploring the contextual relationship. Journal of Business Research, 76, 77-88. https://doi.org/10.1016/j.jbusres.2017.03.010

Stern, P. C., Dietz, T., Abel, T., Guagnano, G. A. y Kalof, L. (1999). A value-belief-norm theory of support for social movements: The case of environmentalism. Human ecology review, 6(2), 81-97.

Stern, P. C. (2000). New environmental theories: toward a coherent theory of environmentally significant behavior. Journal of Social Issues, 56(3), 407-424. https://doi.org/10.1111/0022-4537.00175

Thøgersen, J. (2006). Understanding repetitive travel mode choices in a stable context: A panel study approach. Transportation Research Part A: Policy and Practice, 40(8), 621-638. https://doi.org/10.1016/j.tra.2005.11.004

Tussyadiah, I. P. (2015). An exploratory study on drivers and deterrents of collaborative consumption in travel. In Information and communication technologies in tourism (pp. 817-830). Cham: Springer.

Vione, K. C. (2012). As prioridades valorativas mudam com a idade? Testando as hipóteses de rigidez e plasticidade. Dissertação de Mestrado. Departamento de Psicologia, Universidade Federal da Paraíba, João Pessoa, Brasil.

Wang, Y., Asaad, Y. \& Filieri, R. (2020). What makes hosts trust Airbnb? Antecedents of hosts' trust toward Airbnb and its impact on continuance intention. Journal of Travel Research, 59(4), 686-703. https://doi.org/10.1177/0047287519855135

Wells, W. D. (1975). Psychographics: A critical review. Journal of marketing research, 12(2), 196-213. https://doi.org/10.2307/3150443

Xiao, G. \& Kim, J. O. (2009). The investigation of Chinese conusmer values, consumption values, life satisfaction, and consumption behaviors. Psychology and Marketing, 26(7), 610-624. https://doi.org/10.1002/mar.20291 